\title{
INTRODUCING FORCE TRACEABILITY TO NANOINDENTATION MEASUREMENTS
}

\author{
${ }^{1}$ Radek ŠLESINGER, ${ }^{2}$ Anna CHARVÁTOVÁ CAMPBELL, ${ }^{3}$ Vilma BURŠÍKOVÁ \\ ${ }^{1}$ Czech Metrology Institute, Brno, Czech Republic, EU, rslesinger@cmi.cz \\ ${ }^{2}$ Czech Metrology Institute, Brno, Czech Republic, EU, acharvatovacampbell@cmi.cz \\ 3Masaryk University, Faculty of Science, Brno, Czech Republic, EU, vilmab@physics.muni.cz
}

https://doi.org/10.37904/nanocon.2020.3774

\begin{abstract}
We present a simple and portable setup for basic characterization of force sensors in nanoindentation instruments. The device can provide traceability to mass standards in the milli- and centinewton range. We present its use with a real nanoindentation instrument. Motivated by the result, we conduct a small computational study to demonstrate the effect of sensor measurement error on material properties quantified from nanoindentation measurements.
\end{abstract}

Keywords: Nanoindentation, traceability, force calibration, indentation modulus, hardness

\section{INTRODUCTION}

Various local mechanical properties, notably the indentation modulus $E$ and hardness $H$ of materials can be determined using nanoindentation [1]. Nanoindentation is a form of so-called instrumented indentation technique (IIT), where data evaluation is based on depth and load values as measured by the respective sensors in the instrument. Proper expression of the mechanical quantities then assumes using a calibrated instrument, e.g. as described in ISO 14577-2 standard [2]. However, most instruments are delivered and further operated without any traceable calibration. Although at least basic verification of instrument performance can be performed using reference samples with certified values of hardness or elastic modulus, this is not sufficient for proper processing of load-depth data. As far as is known to the authors, sensor calibration is not actively supported by instrument manufacturers, and users interested in calibrating their instrument have to rely on their own solutions. The possibilities are, however, limited by instrument design and space available for mounting the calibration equipment. When sensor calibration is not available, attention should be paid to the values calculated in measurement evaluation.

\section{FORCE CALIBRATION}

In case of vertical load application, force measurement can be viewed as equivalent to weighing. As maximum loads that can be applied by nanoindentation heads of different instruments vary from units up to hundreds of millinewtons, this renders to measuring in the sub-gram up to decagram range. Given that the gravity of Earth in the instrument location is known, metrological traceability of force can be introduced by means of calibrated weights.

A key component in most special setups for very fine force measurements is a microbalance based on the electromagnetic force compensation principle, see e.g. [3]. Such balances are capable of resolving weights as low as $0.1 \mu \mathrm{g}$ (which roughly corresponds to the load difference of $1 \mathrm{nN}$ ), but their dimensions and weights are often inappropriate for mounting in the indentation instrument. 
Our goal was therefore to construct a device which is compact enough to fit in most instruments. It is built on a commercially available load cell (based on the usual tensometric bridge construction) with a 10-gram range, and a precise $A / D$ converter for voltage readouts, namely:

- a GSO-10 load cell, manufactured by Transducer Techniques, USA, equipped with an extension stem for weight stacking (see Figure 1); the height of the sensor including the extension stem is less than $25 \mathrm{~mm}$;

- a 24-bit A/D converter AD24USB, manufactured by JanasCard, Czech Republic, providing and measuring the excitation voltage for bridge input and measuring the bridge output voltage;

- $\quad$ a set of F1-class calibration weights (nickel-silver lamina weights, stainless steel cylindrical weights allowing easier stacking).

The sensor is mounted on a metal plate for better stability. To achieve better portability and ability to run standalone for extended periods of time, the $A / D$ converter was connected to a single-board computer. $A$ custom software tool was used for reading the digitalized values from the converter, expressed in the usual way in as the ratio of the output and excitation voltage in $\mu \mathrm{V} / \mathrm{V}$.

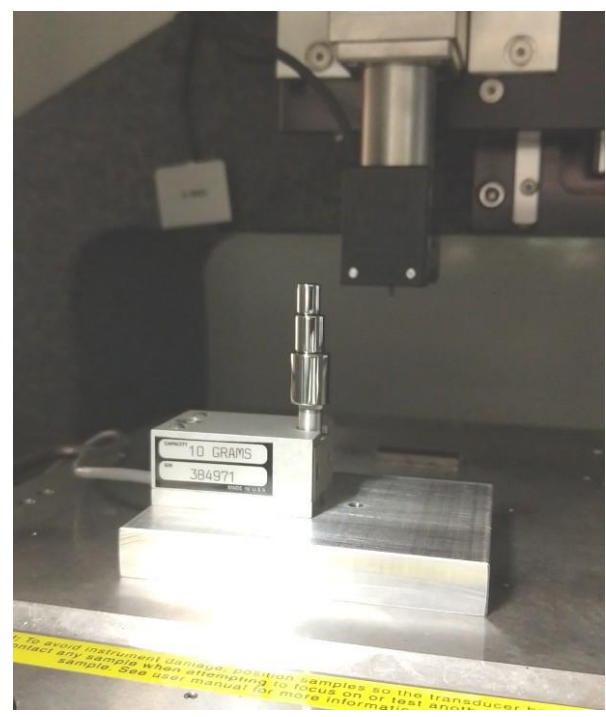

Figure 1 GSO-10 sensor with a stack of calibration weights, mounted in the nanoindentation instrument

Two measurement runs were carried out in the experiment:

1. using the set of calibration weights,

2. using a series of pre-defined loads in the indentation instrument software, calculated from the corresponding weights and the gravity of Earth of $9.80940003 \mathrm{~m} / \mathrm{s}^{2}$ as provided by the Czech Office for Surveying, Mapping and Cadastre for the location Brno, Czech Republic.

Maximum load applied by the instrument was $800 \mathrm{mg}$ due to measurement head limitations. To eliminate the effect of sensor drift during continuous measurement, load applications were interleaved with zero-load periods, so that each load could be evaluated as separate measurement. Although comparisons were carried out using loads as low as $10 \mathrm{mg}$, according to general recommendations, measurements within the lower $1 \%$ of the sensor range should not be taken into account. Unfortunately, no sensor that has a finer range and allows stacking calibration weights is known to the authors, which limits applicability of the current setup to loads of $100 \mathrm{mg}$ and above.

Results of the comparison are presented in Figure 2. They suggest that the instrument in our experiment might systematically overstate its force measurements by approx. $1 \%$. 


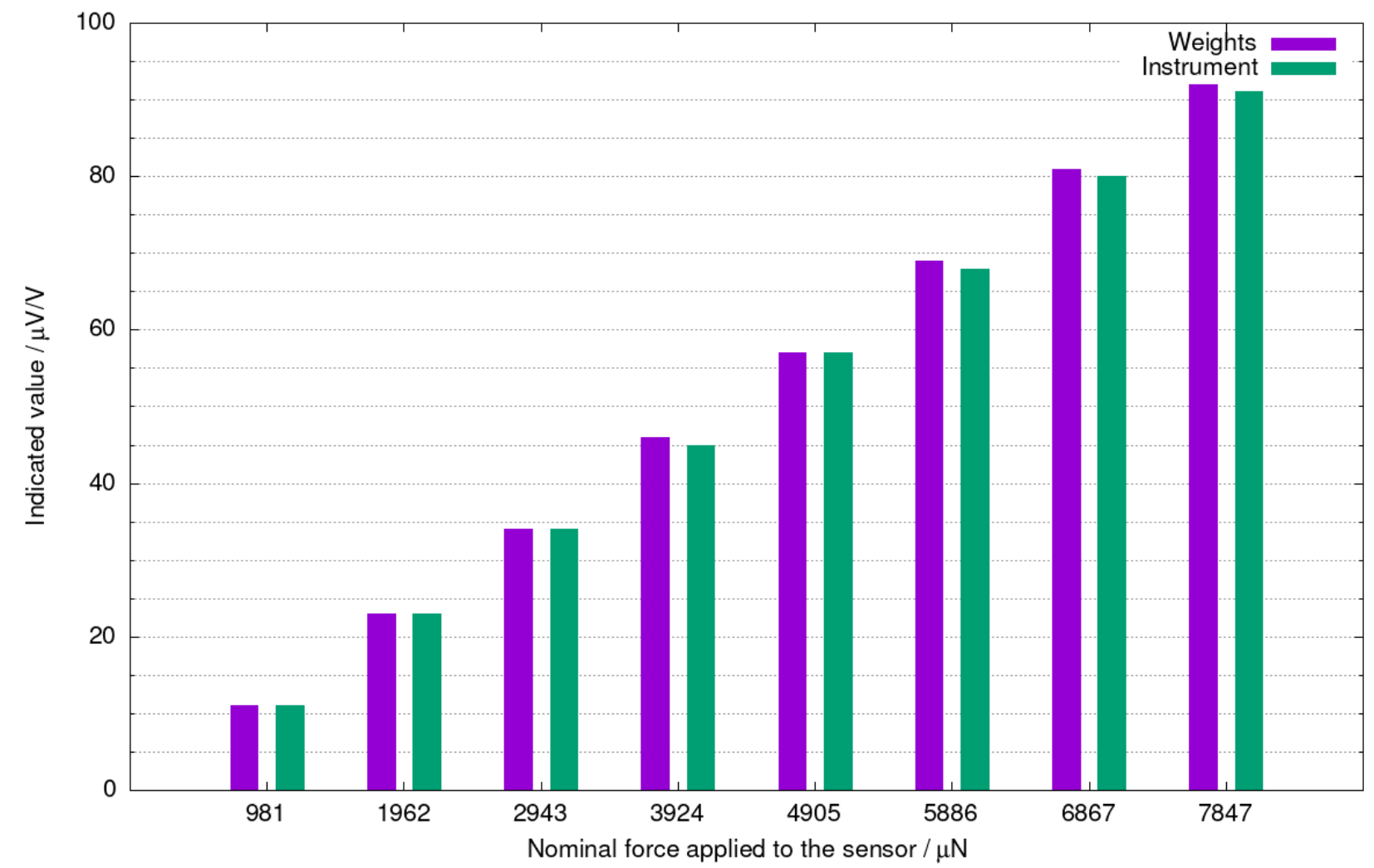

Figure 2 Comparison of indicated values between the loads generated by weights and by the nanoindentation instrument

\section{DATA CORRECTION ACCORDING TO CALIBRATION RESULTS}

Proper analysis of instrumented indentation data involving sensor calibration and related uncertainties remains a major endeavor. Instead, let us demonstrate on a simple example how an inaccurate measurement by the sensors of a nanoindentation instrument can affect the mechanical quantities of main interest as determined by the commonly used method due to Oliver and Pharr [2,4,5].

Based on a small set of measurement data and the elastic modulus of a fused silica reference sample, we determine the contact area function of an indentation tip in the form $A(h)=x^{2}+a x+b x^{1 / 2}+c x^{1 / 4}+d x^{1 / 8}$ using an orthogonal least squares fit. Based on this area function, we evaluate measurements on three different samples: sapphire $\left(\mathrm{Al}_{2} \mathrm{O}_{3}\right.$, load $\left.5 \mathrm{mN}\right)$, copper $(\mathrm{Cu}, 5 \mathrm{mN})$, and polycarbonate $(\mathrm{PC}, 1 \mathrm{mN})$.

Motivated by the results from the previous section, we simulate two basic scenarios of inaccurate sensor measurement:

4. The load sensor overstates the values, and load values have to be reduced by $1 \%$.

5. The depth sensor overstates the values, and depth values have to be reduced by $1 \%$.

The original procedure is then repeated after reducing all load or depth values by $1 \%$, to correct for the measurement error of the respective sensor. Results of this comparison are presented in Table 1. The original (i.e., based on the inaccurate measurement) values of indentation modulus and hardness are denoted $E_{100}$ and $H_{100}$. The "true" values, calculated from the corrected data, are denoted E99L and $H_{99 L}$ (load correction) or $E_{99 D}$ and $H_{99 D}$ (depth correction). The values in parentheses express the relative difference to the uncorrected figures. 
Table 1 Influence of inaccurate measurements of the load or the depth sensor on calculated values of indentation modulus and hardness

\begin{tabular}{|l|l|l|l|}
\hline & $\mathrm{Al}_{2} \mathrm{O}_{3}$ & $\mathrm{Cu}$ & $\mathrm{PC}$ \\
\hline$E_{100} / \mathrm{GPa}$ & 286.97 & 132.70 & 2.70 \\
\hline$H_{100} / \mathrm{MPa}$ & 17351.1 & 1312.1 & 132.7 \\
\hline$E_{99 \mathrm{~L}} / \mathrm{GPa}$ & $287.36(+0.14 \%)$ & $132.76(+0.05 \%)$ & $2.70(+0.00 \%)$ \\
\hline$H_{99 \mathrm{~L}} / \mathrm{MPa}$ & $17564.4(+1.23 \%)$ & $1326.5(+1.10 \%)$ & $133.8(+0.83 \%)$ \\
\hline$E_{99 \mathrm{D}} / \mathrm{GPa}$ & $288.33(+0.47 \%)$ & $133.55(+0.64 \%)$ & $2.72(+0.74 \%)$ \\
\hline$H_{99 \mathrm{D}} / \mathrm{MPa}$ & $17137.3(-1.23 \%)$ & $1300.9(-0.85 \%)$ & $132.1(-0.45 \%)$ \\
\hline
\end{tabular}

While the effect of load measurement error on the indentation modulus is mostly negligible, it directly translates to hardness values (force sensor providing higher than true values results in underestimating the hardness). On the other hand, error of the depth sensor noticeably affects both indentation modulus and hardness (depth sensor providing higher than true values results in underestimating the modulus and overestimating the hardness).

In case that sensor calibration and subsequent proper uncertainty evaluation is not available, we suggest including an uncertainty term due to measurement error in the aggregate budget since such relatively small measurement errors can easily be expected. All the calculations in this section were performed using a free software tool for nanoindentation data processing Niget [6].

\section{CONCLUSION}

A custom setup for measuring forces in the milligram range showed that the force sensor of the evaluated nanoindentation instrument provided slightly, but noticeably inaccurate load values. This should raise attention of users of instrumented indentation technique to the possibility of calibrating their instruments. A computational study demonstrated an apparent influence of sensor measurement errors on the calculated mechanical quantities. In case of uncalibrated instruments, this suggests to expand the uncertainty budget with respective terms to account for assumed errors.

\section{ACKNOWLEDGEMENTS}

This work has been supported by the Czech Science Foundation project GA19-15240S "Multifunctional Nanocomposite Polymer Thin Films with Controlled Surface and Mechanical Properties Deposited in RF Dusty Plasma".

\section{REFERENCES}

[1] FISCHER-CRIPPS, A. C. Nanoindentation. New York: Springer-Verlag, 2004.

[2] ISO/IEC, 14577-2:2015. Metallic materials - Instrumented indentation test for hardness and materials parameters - Part 2: Verification and calibration of testing machines. Geneva: ISO/IEC, International Organization for Standardization, 2015.

[3] SCHLEGEL, C., SLANINA, O., HAUCKE, G., KUMME, R. Construction of a standard force machine for the range of $100 \mu \mathrm{N}-200 \mathrm{mN}$. Measurement. 2012, vol. 45, pp. 2388-2392.

[4] ISO/IEC, 14577-1:2015. Metallic materials - Instrumented indentation test for hardness and materials parameters - Part 1: Test method. Geneva: ISO/IEC, International Organization for Standardization, 2015.

[5] OLIVER, W. C., PHARR, G. M. An improved technique for determining hardness and elastic modulus using load and displacement sensing indentation experiments. Journal of Materials Research. 1992, vol. 7, pp. 1564-1583.

[6] CHARVÁtOVÁ CAMPBELL, A., GROLICH, P., ŠLESINGER, R. Niget: Nanoindentation general evaluation tool. SoftwareX. 2019, vol. 9, pp. 248-254. 